\title{
Las lenguas indígenas y la historiografía de América Latina ${ }^{1}$
}

\author{
Alan Durston
}

\section{RESUMEN}

La historiografía de América Latina está cada vez más consciente del hecho de que el desarrollo de las lenguas indígenas después de la conquista no se puede entender como un proceso lineal de declive, y que en ellas existen fuentes valiosas provenientes de lugares y épocas imprevistas. Hace bastante tiempo que una parte importante de la historiografía del Mé-

1 Este artículo apareció originalmente en inglés («Indigenous Languages and the Historiography on Latin America», Storia della Storiografia, vol. 67, 2015, pp. 5165). Juan Maiguashca, editor del número especial de Storia della Storiografía donde apareció la versión original, me alentó a escribir el artículo, a mejorarlo y a esclarecer varios puntos. También fueron de mucha ayuda las lecturas críticas de Jeremy Mumford, Mark Thurner, Camilla Townsend y Yanna Yannakakis. Capucine Boidin y Bridget Chesterton me orientaron con el apartado sobre Paraguay. Por último, agradezco a Jorge Hidalgo y Olaya Sanfuentes, por la iniciativa de publicar una versión española en Allpanchis, y a los editores de Storia della Storiografia, por autorizar esta versión. La traducción fue realizada por Oscar Patsen. 
xico colonial ha hecho un fuerte uso de las fuentes en lenguas indígenas, y estas han comenzado a tener presencia en la historiografía de otras regiones. Este artículo analiza el tratamiento de las lenguas indígenas y sus fuentes en las historiografías de México, Perú y Paraguay. Sostiene que las nuevas tendencias más prometedoras en este ámbito prestan una mayor atención a la historia social del lenguaje, al uso de las lenguas indígenas por personas no indígenas, y a su utilización en los procesos de construcción nacional.

Palabras clave: historiografia, Latinoamérica, lenguas indígenas, nábuatl, quechua, guaraní

\section{Abstract}

The historiography on Latin America is increasingly cognizant of the fact that the post-conquest development of indigenous languages cannot be understood in terms of a linear process of decline, and that there are valuable sources in these languages from unexpected times and places. An important segment of the historiography on colonial Mexico has long made intensive use of indigenous-language sources, and indigenous languages are beginning to appear on the historiographical radar elsewhere. This article surveys the treatment of indigenous languages and indigenous-language sources in the historiographies on Mexico, Peru, and Paraguay. It argues that the most promising new trends in the field include greater attention to the social history of language, to the use of indigenous languages by nonIndians, and to their use in nation-building processes.

KEYwords: historiography, Latin America, indigenous languages, $\mathrm{Na}$ buatl, Quechua, Guarani 


\section{INTRODUCCIÓN}

LA HISTORIOGRAFía DE AmÉRICA LATINA recién ha comenzado a reflejar la vitalidad posconquista de las lenguas indígenas, vitalidad pasada por alto durante mucho tiempo, a pesar de que existen varios países que hoy tienen mayorías que hablan lenguas indígenas o las han tenido en tiempos recientes. ${ }^{2}$ Solo entre los estudiosos del México colonial se ha desarrollado una verdadera historiografía basada en fuentes en lenguas indígenas. Esta historiografía se focaliza en el centro de México (de habla náhuatl), Oaxaca (zona plurilingüe) y Yucatán (habitada por hablantes de maya yucateco). En estas zonas existe una abundancia excepcional de textos escritos en lenguas indígenas por autores indígenas durante los siglos XVI y XVII, entre los que se incluyen miles de documentos notariales, un tipo de fuente que se encuentra en cantidades importantes como en ningún otro lugar. En los años setenta, una escuela historiográfica que se enfocó en tales fuentes comenzó a surgir bajo el liderazgo del recientemente fallecido James Lockhart, uno de los historiadores de la América Latina colonial más influyentes.

No hace mucho, las lenguas indígenas han logrado un mayor protagonismo en la historiografía de otras áreas de América Latina, especialmente en Perú y Paraguay. Esto es, en parte, resultado de nuevas investigaciones sobre fuentes desconocidas o poco conocidas, especialmente, aquellas del periodo colonial tardío y nacional; también refleja un interés creciente en las preguntas que surgen de la continua vitalidad de las lenguas indígenas: ¿Qué se puede aprender sobre —y de — el vaivén demográfico y social de las lenguas indígenas a través del tiempo? ¿Cómo era el manejo de la comunicación entre hablantes de idiomas diferentes en las sociedades coloniales y

2 Este es el caso de Bolivia, Guatemala, Paraguay y Perú, y quizá, el de otros países. Las lenguas indígenas mantienen una fuerte presencia en los estados sureños de México. 
nacionales? ¿Cuáles fueron los efectos culturales y sociales de la diversidad lingüística y del multilingüismo? ¿Quiénes hablaban lenguas indígenas, en qué contextos y por qué? Lo que Peter Burke (1993) denomina la «historia social de la lengua» — una sociolingüística histórica - ha recibido poca atención en América Latina.

Este ensayo tiene como objetivo identificar las tendencias fundamentales en el uso de fuentes en lenguas indígenas en la historiografía, ${ }^{3}$ y en el estudio de la historia social de las mismas. Mi definición de «historiografía» incluye el trabajo de especialistas en disciplinas afines que están interesados en los procesos de cambio en las sociedades latinoamericanas. No se incluyen las investigaciones con textos no alfabéticos. El principal avance en esta área durante las últimas décadas ha sido el desciframiento de la escritura glífica maya, sin embargo, la mayoría de los textos existentes son inscripciones que datan de siglos antes de la conquista española. ${ }^{4} \mathrm{El}$ quipu andino, o sistema de registro de cuerdas y nudos, es el tema de un floreciente campo de investigación. Ahora se sabe que los quipus se siguieron utilizando en algunas partes de Perú durante el siglo xx. No obstante, existe consenso en que los quipus no registraban información lingüística, por lo tanto, no se puede afirmar que se utilizaban para escribir en lenguas indígenas (Quilter y Urton 2002; Salomon 2004).

El artículo está organizado geográficamente, y se enfoca en las tres zonas donde la documentación en lengua indígena tiene mayor presencia: 1) Mesoamérica, donde existen numerosas fuentes en diversas lenguas, 2) los Andes (principalmente Perú, mi campo de

3 No abarca el uso de las fuentes de autoría indígena en la historiografía: muchos textos históricos de autoría indígena fueron escritos en lenguas europeas y muchos textos en lengua indígena son obra de europeos, criollos y mestizos. Se enfocan los trabajos cuya metodología involucre el estudio directo de textos en lenguas indígenas. Para un estudio importante acerca de los textos indígenas en América Latina, véase Leinhard (1992).

4 Para una descripción general reciente, véase Tedlock (2010). 
especialización), en donde el quechua es el grupo lingüístico mejor representado, y 3) Paraguay (guaranî). ${ }^{5}$ El trabajo de los mexicanistas entrega un marco de referencia para examinar lo que se ha realizado en las otras dos áreas. No pretendo ofrecer un análisis cabal y sistemático de tan vasto campo y los lectores encontrarán muchas omisiones; especialmente en lo que concierne a Mesoamérica, en donde mi informe está restringido a las investigaciones más influyentes publicadas en inglés. Comienzo con algunas acotaciones generales sobre la historia de las lenguas indígenas en Latinoamérica y de las fuentes conocidas.

\section{Las lenguas indígenas de América Latina y SU PRODUCCión ESCRITA}

Las zonas del hemisferio occidental que fueron colonizadas por España y Portugal están entre las de mayor diversidad lingüística del mundo. Mesoamérica llama especialmente la atención, debido al número de grupos lingüísticos diferentes atestados en un área relativamente pequeña. El náhuatl, maya yucateco y mixteco — las tres lenguas mesoamericanas mejor documentadas - pertenecen a tres familias lingüísticas (uto-azteca, maya y otomangue) que, aparentemente, no provienen de ningún tronco común. La región andina (entendida aquí como las zonas de altura y costera de Ecuador, Perú y Bolivia) se muestra menos diversa, con predominio de las familias lingüísticas del quechua y aimara, sin embargo, varias otras lenguas de la región desaparecieron durante el transcurso de los siglos XIX y xx. La diversidad lingüística de las tierras bajas de Sudamérica,

5 Mesoamérica es una región cultural que abarca desde el centro y sur de México hasta el norte de Centroamérica. Las fuentes e investigación vienen preponderantemente de lo que ahora es México; debido a esto, hablaré indistintamente de México y Mesoamérica. 
especialmente la Amazonía, es notoria, aunque aquí los lingüistas han identificado dos familias muy numerosas y extensas — arawako y tupí- que están presentes en toda la región. ${ }^{6}$

Los esfuerzos que se están haciendo para determinar cuándo y cómo ciertas lenguas o grupos lingüísticos lograron expandirse a través de extensas áreas son de gran interés para los historiadores. Un ejemplo de esto es el debate sobre cómo las variantes del quechua se establecieron desde Colombia hasta el noroeste argentino. Un importante cambio en la forma de concebir este tema ocurrió en las décadas de los sesenta y setenta, cuando se refutó el modelo convencional que planteaba que el quechua había nacido en Cusco y expandido con la dominación inca en el siglo xv. En cambio, se propuso que el quechua se había desarrollado primero en la zona central de Perú y luego se propagó hacia el norte y sur del país en diferentes oleadas, principalmente en la época preincaica (Torero 1974). Actualmente, se está perfeccionando este modelo con nuevas investigaciones que ponen mayor énfasis en las transformaciones ocurridas durante los siglos XV y XVI — la época de las conquistas incas y españolas - aunque reconocen que el quechua ya estaba presente en gran parte de las zonas de altura peruanas, tal vez como consecuencia de la expansión del imperio huari a fines del primer milenio de nuestra era (Heggarty y Beresford-Jones 2012; Itier 2013; Kerke y Muysken 2014).

Algunas expansiones se llevaron a cabo después de la llegada de los europeos. Las expediciones conquistadoras, acompañadas de aliados indígenas, nuevas actividades económicas y redes comerciales, migraciones y toda clase de trastornos relacionados con la conquista y la colonización resultaron en el uso de algunas lenguas indígenas en nuevos contextos, y su consecuente transformación.

6 Para estudios lingüísticos regionales, véase Edmonson y Andrews (1984) (Mesoamérica); Adelaar y Muysken (2004) (área Andina); O’Connor y Muysken (2014) (Amazonía). 
En muchas partes de la América Latina colonial existieron lenguas indígenas que servían como lenguas vehiculares, al utilizarse para la comunicación entre indígenas y europeos, y entre indígenas que hablaban idiomas diferentes. A mediados del siglo XVI surgió la expresión lengua general (en portugués língua geral), para referirse a las lenguas indígenas que fueran muy utilizadas (Itier 2013).

Algunas «lenguas generales» ya habían funcionado como vehiculares previo a las conquistas ibéricas —el náhuatl es un claro ejemplo_-, mientras que otras solo parecen haber adquirido esta función posteriormente. Quizá el ejemplo más claro de esto último sea el tupinambá o tupí, una lengua hablada a lo largo de gran parte de la costa brasileña cuando llegaron los primeros europeos. La utilizaron los primeros colonos y sus hijos, y más tarde adquirió las funciones de lengua vehicular en gran parte del interior del país, donde fue llevada por mestizos cazadores de esclavos, colonos y sus aliados indígenas, y misioneros jesuitas. En el siglo XVII, un tipo de tupinambá se convirtió en la lengua oficial de la Amazonía portuguesa. La población autóctona, que originalmente hablaba una variedad de lenguas diferentes, la utilizaba para comunicarse entre sí y con los portugueses. Durante el proceso, la lengua sufrió una transformación, y la variedad resultante es conocida por los estudiosos como língua geral amazônica (ñengatú moderno). Para ese entonces, los hablantes originales del tupinambá prácticamente habían desaparecido; sólo sobrevivió su lengua en una nueva forma. ${ }^{7}$

En aquellas zonas donde España y Portugal establecieron una fuerte presencia durante el siglo XVI, los misioneros pronto adaptaron sus variantes nacionales del alfabeto latino para escribir las «lenguas generales», así como también algunas de las menos conocidas. Se establecieron imprentas en Ciudad de México y Lima, en parte, con el propósito de publicar gramáticas, diccionarios y libros

7 Acerca de la língua geral amazônica, véase Moore (2014: 108-142). Para una perspectiva histórica acerca del desarrollo de Tupinambá, véase Lee (2014: 143-167). 
de catequesis en lenguas indígenas para el uso del clero. En Mesoamérica, las élites nativas escribieron sus lenguas alfabéticamente con múltiples fines, entre los que se incluían la mantención de los registros administrativos, el registro de las historias de la comunidad e incluso la tradición religiosa precristiana. ${ }^{8}$ Sus contrapartes andinas fueron menos prolíficas: existe un pequeño corpus de cartas y peticiones de autoría indígena en quechua que datan de los siglos XVI y XVII, además de los libros de catequesis y otras obras escritas por los sacerdotes. ${ }^{9}$ Fuera de las zonas andina y mesoamericana apenas se tienen referencias de escritos en lengua indígena de la época colonial que no hayan sido realizados por misioneros. La principal excepción es el guaraní, en Paraguay. Por razones que se discutirán más adelante, los líderes guaraníes comenzaron a producir documentación administrativa en español y guaraní a mediados del siglo XVIII; previo a ello, el guaraní escrito había sido fundamentalmente el instrumento de los misioneros jesuitas (Melià 1992, 2011).

Las reformas borbónicas de la segunda mitad del siglo XVIII, seguidas por las guerras de independencia de las décadas de 1810 y 1820, y la caótica construcción de las repúblicas latinoamericanas, se ven reflejadas en cambios significativos en la naturaleza del registro en lenguas indígenas. La política borbónica era hostil hacia las lenguas autóctonas, y una cédula real de 1770 incluso llamó a su erradicación. ${ }^{10} \mathrm{El}$ uso escrito de las lenguas indígenas comenzó

8 Para descripciones generales y antologías recientes, véase Edmonson y Andrews (1985); Restall, Sousa y Terraciano (2005); Tedlock (2010); Lockhart, Sousa y Wood (2007).

9 Para discusiones generales acerca de escritos coloniales en quechua, véase Mannheim (1991: \6) y Durston (2008). Para una antología de literatura misionera colonial en quechua, véase Taylor (2003). Para una antología de literatura quechua de Cusco, véase Itier, Nieto Degregori y Vargas Prado (2012). También son útiles los trabajos recopilados en Pillsbury (2008), especialmente Harrison (2008: 217-234) y Dedenbach-Salazar Sáenz (2008: 235-264).

10 «Real Cédula para que en los Reinos de Las Indias se Destierren los diferentes idiomas de que se usa, y solo se hable el Castellano» (Solano 1991: 257-261). 
a disminuir en diferentes momentos entre mediados del siglo XVII y comienzos del xIx, debido a una serie de factores, en especial, la castellanización y el declive sociopolítico de las élites indígenas (Lockhart 1992; Restall, Sousa y Terraciano 2005: 15-17). Pero, aunque se escribiera menos, aparecieron nuevos géneros durante la era de las revoluciones. Los líderes de la independencia y las fuerzas realistas publicaron proclamaciones en varias lenguas indígenas (Laughlin 2003; Morris 2007; Durston 2011 y Melià 2011). Las convulsiones de comienzos del periodo nacional (aproximadamente de 1820 a 1880) están asociadas al florecimiento de la escritura política en maya yucateco (durante la Guerra de Castas, 1847-1901) y guaraní (durante la Guerra de la Triple Alianza, 1864-1870). ${ }^{11}$

Nuevas circunstancias afectaron la escritura en lenguas indígenas a fines del siglo XIX y principios del xx. Este fue un espacio de tiempo en que muchos estados latinoamericanos estaban expandiéndose a nuevos territorios sobre los cuales anteriormente no habían tenido un control real, conquistando y, a menudo, exterminando la población indígena. Este proceso se llevó a cabo a gran escala en la Amazonía y el Cono Sur, pero sucedió en toda Latinoamérica, y permitió una nueva ola de evangelización católica y la llegada de los primeros etnógrafos modernos a estas zonas. Si bien el proceso fue devastador para los pueblos originarios involucrados, un efecto colateral fue que ellos — y sus lenguas — ingresaron por primera vez en el registro alfabético, o, al menos, lo hicieron en mayor medida que en el pasado (Salomon 1999).

Los mapuches del sur de Chile y Argentina constituyen un ejemplo notable. Los únicos textos del periodo colonial en mapudungún, la lengua mapuche, son obras misioneras: los mismos mapuches tenían poca necesidad de escribir, debido a que lograron

11 Acerca de los documentos de la Guerra de Castas en maya yucateco, véase Bricker (1981); para textos en guaraní de la Guerra de la Triple Alianza, véase http://www.staff.uni-mainz.de/lustig/guarani/ 
permanecer independientes hasta la década de 1880. Durante las primeras décadas del siglo xx, un corpus de textos en mapudungún, entre los cuales se incluían biografías de caciques mapuches, fue publicado bajo la supervisión de misioneros y etnógrafos. Aun cuando se sustentaban en la oralidad, estos testimonios fueron escritos por la primera generación de mapuches alfabetizados, que eran, en su mayoría, hijos de los últimos caciques independientes. La colección y publicación de testimonios mapuches contó con la supervisión y promoción de misioneros y etnógrafos chilenos y europeos, so pretexto de dejar registro de una cultura en vías de extinción para la ciencia y para ayuda del proceso civilizador. No obstante, se ha sugerido que los intelectuales mapuches que trabajaban con ellos estuvieran comprometidos paralelamente con el proyecto de escribir la historia del pueblo mapuche (Pavez Ojeda 2003).

El periodo de cambio de siglo también fue testigo del inicio de un fenómeno que traería múltiples consecuencias para la escritura en lenguas indígenas durante la primera mitad del siglo xx: el surgimiento del nacionalismo y regionalismo indigenistas. Los intelectuales latinoamericanos desarrollaron un interés por las lenguas indígenas como parte de una reacción nativista contra la creciente influencia de Estados Unidos. Esto no significó que existiera una gran cantidad de escritos en lenguas indígenas realizados por los intelectuales de las capitales latinoamericanas, quienes eran, generalmente, monolingües en la lengua dominante. Sin embargo, al mismo tiempo que el nacionalismo nativista iba en aumento, las élites regionales que sí hablaban lenguas indígenas reaccionaban ante la creciente influencia de los estados centrales, y ante la integración económica y territorial, lo que significó una explosión literaria en lenguas indígenas.

Un buen ejemplo de esto se puede encontrar en el pueblo de Juchitán, en el sur de México, donde se habla zapoteco. Howard Campbell (1994: 120-127) señala que los intelectuales locales comenzaron a escribir sobre - y en- zapoteco cuando migraron a 
Ciudad de México, para acceder a mejores oportunidades políticas, educacionales y laborales. La primera migración se produjo a fines del siglo XIX y, en mayor escala, después de la Revolución Mexicana (1910-1920). En la década de los treinta, los intelectuales de Juchitán fundaron una academia de lengua zapoteca y publicaron una revista literaria (escrita en español y zapoteco) en Ciudad de México. La exposición al contexto metropolitano durante la era indigenista les hizo tomar conciencia de su particularidad y generó un deseo por promover una identidad étnica/regional.

Durante este mismo periodo, los intelectuales de la sierra peruana, especialmente de Cusco y Ayacucho, escribían obras de teatro y poesía en quechua (Itier 1995a, 2000; Durston 2014). Estos autores difieren en algunos aspectos cruciales de sus contrapartes de Juchitán: no se identificaban como indígenas y tendieron a tener una relación mucho más antagónica con la capital nacional. A pesar de esto, también tenían mucho en común: pertenecían a élites provinciales bilingües que encontraron un capital cultural en sus «lenguas maternas» indígenas, a la par que eran integrados, o subordinados, a la sociedad nacional metropolitana.

Además del florecimiento de la literatura en lenguas indígenas, la primera mitad del siglo xx fue testigo de la aparición de los primeros materiales para la educación pública. Los catecismos y otros textos para la enseñanza religiosa eran, por supuesto, abundantes en muchas lenguas desde el siglo XVI, pero fue recién a comienzos del siglo xx que se propuso que el Estado debía publicar textos de educación básica en lenguas indígenas. El gobierno revolucionario de México lideró esta tendencia, publicando libros en distintos idiomas y solicitando los servicios de lingüistas estadounidenses, muchos de los cuales trabajaban en el Instituto de Lingüística de Verano, una organización misionera evangélica, para desarrollar materiales educativos y ortográficos para las lenguas menos conocidas (Dawson 2004; Hartch 2006). En México y Perú, y probablemente en otros lugares, la época indigenista coincidió con periodos de revueltas 
rurales y movimientos reformistas que procuraron movilizar o incorporar al campesinado indígena, dando como resultado el resurgimiento del tipo de propaganda política en lenguas indígenas que floreció durante las guerras de independencia (León-Portilla 1978; Durston [En prensa]).

No intentaré hacer un repaso de la escritura en lenguas indígenas en la segunda mitad del siglo xx, pero se pueden hacer algunas generalizaciones. La mayor parte de este material consta de transcripciones de narraciones orales realizadas por antropólogos y lingüistas tras grabar en terreno a informantes indígenas. La aparición de la grabadora magnetofónica significó la publicación de miles de textos, no obstante, la escritura directa en lenguas indígenas decayó a mediados del siglo xx. En el caso del quechua, y quizá otras lenguas, esta caída está relacionada con el declive de las élites provinciales bilingües y sus proyectos intelectuales (Itier 2002). Este también fue un periodo de urbanización acelerada en toda Latinoamérica con el consecuente declive demográfico de las lenguas indígenas: entre 1940 y 1993, el porcentaje de peruanos que hablaba quechua cayó de 47 \% a 18 \% (Itier 2002). Sin embargo, el resurgimiento de las identidades indígenas que ha ocurrido en toda América Latina durante las últimas dos o tres décadas está cambiando rápidamente el panorama de la escritura en lenguas autóctonas. ${ }^{12}$

\section{Mesoamérica y la NueVa filología}

Cuando en la década de los setenta James Lockhart inauguró lo que más tarde se conocería como la «Nueva filología», ya existía una tradición de estudios antropológicos y lingüísticos basados en textos coloniales en náhuatl y lenguas mayas. Estos estudios se

12 Algunos proyectos de escritura indígena de fines del siglo xx se discuten en el volumen preparado por Florencia Mallon (2012). 
enfocaban en la interpretación de las cosmovisiones mesoamericanas preconquista a partir de los «códices» alfabéticos —extensos manuscritos de naturaleza histórica, etnográfica o religiosa-. ${ }^{13}$ Lockhart y sus estudiantes, en cambio, privilegiaron el abundante y poco conocido material de archivo en náhuatl —documentos como testamentos, ventas de tierras y registros municipales- para escribir la historia del México colonial desde un punto de vista indígena. La riqueza de estas fuentes no solo reside en el hecho de que estén escritas en lenguas autóctonas, sino también en el hecho de ser registros internos detallados y realizados por autoridades y escribas indígenas. Durante los setenta y ochenta, Lockhart, sus estudiantes y asociados llevaron a cabo una serie de estudios importantes de pueblos y provincias nahuas tomando principalmente como referencia dichas fuentes. En una segunda etapa de la Nueva filología, los estudiantes de Lockhart repitieron exitosamente el proceso de ubicar y analizar cientos o miles de documentos en lengua indígena fuera de la zona nahua. Los principales ejemplos son el trabajo de Matthew Restall en Yucatán (con el maya yucateco) y el de Kevin Terraciano en Oaxaca (con el mixteco). ${ }^{14}$

Su trabajo es, sobre todo, historia social con especial énfasis en las formas indígenas de organización sociopolítica, y su vitalidad y resiliencia bajo el dominio español. Lockhart junto con sus estudiantes y colaboradores han demostrado que las fuentes en lenguas indígenas a menudo muestran una realidad muy distinta a la documentación española, y sugieren una mayor continuidad con la sociedad preconquista. El término «Nueva filología» pareciera ser una designación extraña para esta historiografía. Este término ori-

13 Por ejemplo, The Book of Chilam Balam of Chumayel, editado y traducido por Ralph L. Roys (1933). Véase también Bernardino de Sahagún (1950-1982); León-Portilla (1959); Edmonson (1971).

14 Para discusiones historiográficas de la Nueva filología, véase Restall (2003) y Lockhart, Sousa y Wood (2007). Las monografías representativas incluyen los trabajos de Lockhart (1997) y Terraciano (2001). 
ginalmente establecía un contraste entre el trabajo de Lockhart y sus estudiantes, respecto del trabajo anterior con fuentes en lenguas mesoamericanas (Lockart, Sousa y Wood 2007: 8). Actualmente existe una abundante historiografía que utiliza fuentes en lengua indígena para investigar temas de sexualidad, género y familia, religión local y escritura histórica en la Mesoamérica colonial, entre otras cosas. ${ }^{15}$ No queda claro si esta historiografía debería clasificarse como Nueva filología, y parece mejor restringir el término a estudios que utilicen grandes cantidades de documentación de archivo en una lengua indígena para producir historias sociales generales, siguiendo las pautas establecidas por Lockhart.

Una parte importante de la Nueva filología posee una innegable naturaleza filológica. Se ha dedicado mucho esfuerzo a ediciones textuales, y los trabajos representativos contienen capítulos sobre lengua y escritura. ${ }^{16}$ Lockhart (1997) y Terraciano (2001) han realizado análisis fascinantes acerca del desarrollo de los sistemas de escritura, demostrando cómo la escritura alfabética coexistía y se entremezclaba con elementos pictográficos indígenas antes de que fueran reemplazados a fines del siglo XVI. Lockhart (1991) y algunos de sus estudiantes también han intentado correlacionar la creciente presencia de los préstamos del español en textos en lengua indígena con el cambio cultural y social de larga duración, entendido como un proceso de hispanización. Estos esfuerzos han arrojado muchas observaciones significativas, no obstante, el método no ha sido imitado ampliamente debido a su énfasis en «etapas» teleológicas y a su enfoque casi exclusivo en los préstamos léxicos como forma de cambio lingüístico, sin mencionar la dificultad para encontrar el conjunto de documentación necesario para realizar este tipo de seguimiento. La Nueva filología no ha puesto tanta atención al cambio semántico (la transformación del significado de las palabras in-

15 Véase Pizzigoni (2012); Sigal (2000, 2011); Tavárez (2011), y Townsend (2010).

16 Véase Anderson, Berdan y Lockhart (1976); Lockhart, Berdan y Anderson (1986). 
dígenas). Las profundas transformaciones semánticas e ideológicas en lenguas y culturas indígenas, producto de la conversión religiosa y las instituciones jurídico-administrativas españolas, recién se están convirtiendo en temas importantes en la historiografía mesoamericana colonial. Los trabajos de dos antropólogas, Louise Burkhart (1989) y Susan Kellogg (1995), sobre la evangelización y sobre el derecho, respectivamente, constituyen importantes excepciones. ${ }^{17}$

Concluiré esta parte con algunas reflexiones sobre trabajos recientes que ponen a las lenguas mesoamericanas en el centro de la atención de manera distinta de la Nueva filología. Hay ahora una mayor consciencia en cuanto a que las lenguas mesoamericanas no solo se resistieron ante la arremetida hispana, sino que se expandieron y fueron transformadas por nuevos contextos y usos. También se ha puesto más atención a entender cómo el dominio colonial dio lugar a la transformación de las relaciones entre distintas lenguas indígenas, y a los factores que determinaron la elección de lengua en contextos tales como el derecho, la administración y evangelización. Un libro escrito por el antropólogo William Hanks analiza lo que él denomina «maya reducido», el maya yucateco occidentalizado de los textos de la época colonial. Hanks afirma que redes de conceptos claves fueron introducidas o transformadas como parte de los proyectos coloniales de formación de pueblos (congregación) y evangelización (Hanks 2010). En 2012, la revista Ethnohistory dedicó un número especial a los usos del náhuatl en contextos coloniales plurilingües y multiétnicos: como lengua vehicular, como lengua introducida en nuevos ámbitos como resultado de las conquistas españolas, y como lengua usada por el clero y por otros no indígenas. La revista pone un novedoso énfasis en la movilidad y maleabilidad del náhuatl. En la introducción, Yanna Yannakakis (2012: 673) plan-

17 La misma Burkhart junto con Barry D. Sell (2004-2009) han realizado un trabajo de estudio y edición de un corpus extenso de obras de teatro en náhuatl con temáticas cristianas del periodo colonial. 
tea el tema del plurilingüismo: «¿Cómo empleaban los súbditos de la colonia distintas lenguas en la vida cotidiana, y por qué usaban una lengua en vez de otra?». No es coincidencia que esta interrogante haya sido planteada por una especialista en la zona de Oaxaca, donde el mixteco, zapoteco, náhuatl y lenguas menos conocidas como el chocho podían coexistir en una sola comunidad. Está surgiendo una historiografía distintiva que explora las condiciones del exuberante plurilingüismo de los archivos oaxaqueños. ${ }^{18}$

Son raras las investigaciones históricas sobre el periodo republicano que involucren las lenguas mesoamericanas y sus fuentes. ${ }^{19}$ En su libro 2000 Years of Mayan Literature (2010), Dennis Tedlock dedica un epílogo de apenas cuatro páginas a los siglos XIX y xx, donde afirma que las fuentes de ese espacio de tiempo son de difícil acceso y que los estudiosos de los mayas se han inclinado hacia textos más antiguos (Tedlock 2010: 399-402). Si bien existen estudios importantes de textos políticos en lenguas mesoamericanas de los siglos XIX y xx, ${ }^{20}$ pareciera que aún faltan desarrollar investigaciones exhaustivas y debates sobre estas fuentes y la historia social de las lenguas indígenas en la época posindependencia.

\section{Los Andes}

Mi análisis sobre la historiografía andina está limitado a las investigaciones relacionadas con el quechua, por lejos, la lengua andina mejor documentada. ${ }^{21} \mathrm{El}$ estudio de los textos quechuas colo-

18 Véase Swanton (2008); Doesburg y Swanton (2011); Yannakakis y SchraderKniffki (2014).

19 No hace falta decir que existe una literatura etnográfica abundante que utiliza fuentes en lengua indígena, en su mayoría oral, recopiladas en el siglo xx.

20 Véase León-Portilla (1959); Bricker (1981: 』 4, apéndice A); Laughlin (2003); Morris (2007).

21 El quechua es, en realidad, una familia de lenguas estrechamente emparentadas, no obstante, lo que Mannheim (1991) llama «quechua sudperuano» predomina 
niales tuvo un comienzo tardío respecto a los textos mesoamericanos. Tomemos como ejemplo la historia editorial del Manuscrito de Huarochirí, un compendio único acerca de la tradición religiosa de la provincia de Huarochirí, escrito por un noble indígena alrededor de 1608. Aunque la riqueza de este texto como fuente sobre la cultura prehispánica es comparable a los códices mesoamericanos más famosos, y pese a que su existencia se ha conocido desde el siglo XIX, la primera traducción directa al español recién fue publicada en 1966 (Arguedas y Duviols 1966), y no fue sino hasta 1991 que apareció una traducción al inglés (Salomon y Urioste 1991). Las investigaciones básicas sobre lingüística histórica quechua no se iniciaron sino hasta la década del sesenta (Cerrón-Palomino 1987, capítulo 3).

Las investigaciones sobre el quechua destacan por la atención que han prestado al uso y la transformación de la lengua por agentes no indígenas. Casi todos los textos existentes en quechua que datan de los siglos XVI y XVII, incluyendo aquellos de autoría indígena, emplean o están fuertemente influenciados por un modelo de escritura desarrollado por la Iglesia con fines de evangelización (Mannheim 1991; Cerrón-Palomino 1992; Durston 2007). Por lo tanto, un objetivo clave de la filología quechua desde la década de los setenta ha sido entender las transformaciones semánticas impulsadas por el clero español, con el fin de hacer un mejor uso de las fuentes coloniales (véase p. ej. Taylor 1999, 2000). Actualmente existe una literatura considerable acerca de los usos misioneros del quechua durante el periodo colonial, y los debates sobre traducción han repercutido en la historiografía sobre religión andina colonial. ${ }^{22}$

El uso del quechua como medio de propagación de identidades regionales y nacionales por parte de las élites no indígenas ha sido un tema central en el estudio de la literatura de fines de la colonia y del periodo nacional. Un lenguaje literario purista y arcaizante,

en el registro escrito.

22 Véase MacCormack (1985); Estenssoro Fuchs (2003); Durston (2007); Dedenbach-Salazar Sáenz (2013); Stanfield-Mazzi (2013); Harrison (2014). 
imaginado como el quechua de los incas, comenzó a desarrollarse en el teatro cusqueño del siglo XVIII, teatro que es interpretado por Bruce Mannheim (1991: 71-74) como la expresión de una forma de patriotismo criollo que existía entre la élite cusqueña. ${ }^{23}$ Buena parte de la literatura de la guerra de independencia utiliza este lenguaje; los folletos impresos por los líderes patriotas, a menudo estaban escritos para que sonaran como salidos de la boca de los incas. Algunos de estos textos se refieren explícitamente al quechua como una base para la identificación nacional (Itier 1995b; Durston 2011). $\mathrm{El}$ «quechua incaico» está representado más extensamente en un corpus de teatro histórico escrito a comienzos del siglo xx para expresar las reivindicaciones de la élite cusqueña que, como heredera de los incas, aspiraba al liderazgo nacional. El estudio de estas obras que realizara César Itier incluye ediciones críticas de cinco obras claves y pareciera ser el estudio más exhaustivo de un corpus en lengua indígena del periodo nacional (Itier 1995a, 2000). Es mencionado frecuentemente por los estudiosos de la historia cultural andina del siglo xx. Itier (2000: 87) hace hincapié en que el carisma de la lengua de los incas era una espada de doble filo: el ímpetu de «cultivar» un quechua presuntamente arcaico y puro ha mermado su posición como lengua de comunicación polivalente.

Una compilación reciente cuyo propósito era reunir a lingüistas e historiadores que trabajan en los Andes demuestra las posibilidades para un intercambio fecundo entre la filología quechua y la historia andina (Heggarty y Pearce 2011). También ejemplifica el principio de que la historia posconquista de las lenguas indígenas no se puede entender en términos de declive lineal. Un capítulo del historiador Adrian Pearce sostiene que el quechua creció demográficamente a mediados del siglo xIx. La inestabilidad política y el retroceso económico que se produjo después de la independencia favoreció un re-

23 César Itier (1995b) tiene una concepción diferente acerca del contexto social de estas obras, argumentando que estaban dirigidas a una élite indígena urbana. 
surgimiento de la población indígena cuyo correlato lingüístico pudo haber sido la retirada del español, de manera que, en la sierra sudperuana, el quechua se convirtió en el medio principal de comunicación y expresión, incluso entre las élites urbanas (Pearce 2011). La evidencia es anecdótica, debido a que no existe información censal, sin embargo, el cuadro que presenta Pearce de una élite quechuizada en la sierra sudperuana del siglo xIx es convincente y de evidente importancia para la historia cultural y social de la región. ${ }^{24}$

\section{Paraguay}

La literatura cristiana en guaraní, producida por las misiones jesuitas en Paraguay, ha sido objeto de estudio por algún tiempo, comenzando por la tesis doctoral de Bartomeu Melià de 1969, La création d'un langage chrétien dans les réductions des guarani au Paraguay, reeditada recientemente en español (Melià 2003). Este bien pudiese ser el primer estudio exhaustivo de un esfuerzo misionero por desarrollar una variedad cristiana de una lengua amerindia. Los textos guaraníes coloniales de naturaleza política y administrativa — en especial cartas y peticiones - solo han atraído la atención últimamente, quizá porque datan de periodos posteriores. El hecho de que existan centenares de documentos de este tipo es una gran sorpresa (Melià 2011).

Eduardo Neumann ha estudiado las circunstancias que llevaron a la élite guaraní a comenzar a escribir dichos textos a mediados del siglo XVIII. Cuando los jesuitas comenzaron a perder el control de las misiones, los caciques guaraníes tuvieron que establecer una relación con autoridades de la Corona que estaban geográficamente distantes, debido a la ausencia de una burocracia laica local bien

24 La hipótesis de Pearce (2011) es apoyada por el hecho de que, en 1940, el monolingüismo español era prácticamente inexistente en una ciudad serrana importante como Ayacucho (Durston 2014). 
desarrollada, como la que existía en los Andes y Mesoamérica. Otra característica del contexto paraguayo fue el hecho de formar parte de una frontera militar muy inestable: las misiones estaban bajo el ataque constante de grupos indígenas independientes y los guaraníes requerían de ayuda de las autoridades coloniales para coordinar acciones defensivas. Por todas estas razones, los caciques guaraníes necesitaban la escritura para comunicarse con las autoridades españolas, enfatizar su lealtad a la Corona e informar los servicios prestados, especialmente, aquellos de tipo militar. Aunque muchos de los escribanos guaraníes escribían en español, también encontraron conveniente hacerlo en guaraní a causa de la existencia de la tradición escrita jesuita (Neumann 2007, 2014).

Capucine Boidin (2014) está estudiando las traducciones al guaraní de cartas y decretos emitidos por los líderes de la independencia rioplatense en la década de 1810, haciendo hincapié en el desarrollo de conceptos políticos básicos. ${ }^{25} \mathrm{Si}$ bien Boidin sitúa estos documentos en relación con la literatura política guaraní de periodos anteriores y posteriores, también subraya que el desarrollo de un «lenguaje político moderno» en guaraní formaba parte de un proceso global. La introducción de nuevos conceptos en guaraní, tales como las nociones liberales de nación o ciudadanía, no se pueden ver como casos aislados: el vocabulario político español estaba experimentando un proceso similar de transformación en esta época. Un proyecto de investigación encabezado por Boidin e Itier ha emprendido un estudio comparativo acerca de cómo estos procesos se ven reflejados en los escritos en quechua, guaraní y otras lenguas sudamericanas durante la época de la independencia. ${ }^{26}$

El interés por la prensa en idioma guaraní durante la guerra de la Triple Alianza (1864-1870) también es un fenómeno reciente,

25 Ver también Melià (2011).

26 El proyecto LANGAS (Lenguas Generales de América del Sur) está desarrollando un repositorio de textos en línea con un sofisticado motor de búsqueda (http:/ / www.langas.cnrs.fr) y ha organizado una serie de talleres internacionales. 
pero la investigación está avanzando con rapidez. Wolf Lustig está realizando una compilación en línea de artículos de diarios de esa época en idioma guaraní, haciéndolos disponibles en facsímiles y transcripciones con traducciones. ${ }^{27}$ Esta prensa en guaraní solo duró mientras hubo conflicto: no existió antes de la guerra y desapareció cuando esta finalizó. Sin embargo, dio como resultado una construcción duradera del guaraní como lengua guerrera, consolidando su importancia fundamental en la identidad nacional paraguaya (Lustig 2007; Whigham 2012). El otro gran conflicto internacional de Paraguay, la Guerra del Chaco (1932-1935), no generó prensa en guaraní, aunque condujo a un resurgimiento del guaraní como lengua nacional, que se vio reflejado, entre otras cosas, en la aparición de poesía y teatro nacionalistas (Chesterton 2013: 10, \ 6-7). Existe, por consiguiente, una correlación característica y persistente entre el guaraní escrito y la guerra.

\section{Conclusión}

El creciente interés por el periodo nacional, por el uso de las lenguas autóctonas por autores no indígenas y por las transformaciones que experimentaron estas lenguas al ocupar nuevos espacios y funciones están entre las tendencias más prometedoras en este campo. Estas tendencias amplían la variedad de textos en lenguas indígenas que son tomados en cuenta como fuentes históricas y tienen gran potencial para entrecruzarse con algunas de las inquietudes más importantes en la historiografía latinoamericana. Este es particularmente el caso del estudio de las lenguas indígenas como portadoras de nacionalismo e ideologías políticas modernas, que puede iluminar los debates sobre cultura y participación políticas en la América Latina de los siglos XIX y xx. Existe una extensa his-

27 Véase http://www.staff.uni-mainz.de/lustig/guarani/ 
toriografía sobre la participación del campesinado indígena en los procesos de construcción de la nación, sin embargo, esta literatura rara vez se pregunta cómo se expresaban conceptos claves y discursos en lenguas indígenas.

Resulta un tanto inesperado que estas tendencias estén más desarrolladas en el estudio del quechua y el guaraní que en la literatura mesoamericanista, más antigua y mucho más abundante. Es precisamente la escasez de fuentes tempranas y de textos de autoría indígena lo que ha obligado a los estudiosos del guaraní y las lenguas andinas a poner mayor atención en las fuentes tardías, y en las de autoría no indígena. Por otro lado, la gran cantidad de fuentes tempranas en lenguas mesoamericanas hizo que se diera un mayor énfasis a las continuidades desde la época preconquista y al desarrollo de metodologías que requirieran grandes cantidades de textos; en otras palabras, enfoques que no son fácilmente aplicables a los periodos posteriores o a otras partes de Latinoamérica.

Las peculiares historias del quechua y el guaraní también han fomentado determinadas líneas de investigación. Las dramáticas y poco comprendidas expansiones y contracciones del quechua, así como su fuerte presencia entre poblaciones mestizas y criollas, han suscitado el interés de los quechuólogos por la historia social de la lengua. La fuerza demográfica del guaraní, sumada a las movilizaciones militares a gran escala que caracterizan la historia moderna del Paraguay, han dado como resultado que esta lengua tenga una importante presencia escrita durante los periodos de conflicto. El lenguaje político de fines de la Colonia y del periodo republicano es ahora el tema principal en los estudios guaraníes. Hacer hincapié en la peculiaridad de estas historias y de estos archivos, sin embargo, no es un argumento para excepcionalismos regionales. Temas que son inevitables para los estudiosos del quechua o el guaraní son a menudo relevantes para investigar otras lenguas, que también experimentaron resurgimientos tardíos y adquirieron un protagonismo especial durante los tiempos de convulsión y cambio político. 


\section{REFERENCIAS}

AdelaAr, Willem F. H. y Pieter C. Muysken

2004 The Languages of the Andes. Cambridge y Nueva York: Cambridge University Press.

Anderson, Arthur J. O., Frances Berdan y James Lockhart (eds.)

1976 Beyond the Códices: The Nabua View of Colonial Mexico. Berkeley y Los Ángeles: University of California Press.

Arguedas, José María y Pierre Duviols (eds.)

1966 Dioses y hombres de Huarochiri. Edición bilingüe. Narración quechua recogida por Francisco de Ávila [¿1598?]. Lima: Museo Nacional de Historia e Instituto de Estudios Peruanos.

BoIdin, Capucine

2014 «Textos de la modernidad política en guaraní (1810-1813)». En: Corpus: Archivos Virtuales de la Alteridad Americana, vol. 4, n. 2. Publicación electrónica [en línea]: <http://corpusarchivos.revues. $\operatorname{org} / 1322>$

BRICKER, Victoria Reifler

1981 The Indian Christ, the Indian King: The Historical Substrate of Maya Myth and Ritual. Austin: University of Texas Press.

Burke, Peter

1993 «The Social History of Language». En: Peter Burke. The Art of Conversation. Ithaca: Cornell University Press, pp. 1-33.

BURKHART, Louise M.

1989 The Slippery Earth: Nahua-Christian Moral Dialogue in Sixteenth-Century Mexico. Tucson: University of Arizona Press.

BurkHart, Louise M. y Barry D. Sell (eds.)

2004-2009 Nabuatl Theater. 4 vols. Norman: University of Oklahoma Press.

Campbell, Howard

1994 Zapotec Renaissance: Ethnic Politics and Cultural Revivalism in Southern Mexico. Albuquerque: University of New Mexico Press. 
Cerrón-Palomino, Rodolfo

1987 Lingüística quechua. Cusco: Centro de Estudios Regionales Andinos Bartolomé de Las Casas.

1992 «Diversidad y unificación léxica en el mundo andino». En: Juan Carlos Godenzzi (ed.). El quechua en debate: Ideología, normalización y enseñanza. Cusco: Centro de Estudios Regionales Andinos Bartolomé de Las Casas, pp. 205-235.

Chesterton, Bridget María

2013 The Grandchildren of Solano López: Frontier and Nation in Paraguay, 1904-1936. Albuquerque: University of New Mexico Press.

Dawson, Alexander S.

2004 Indian and Nation in Revolutionary Mexico. Tucson: University of Arizona Press.

Dedenbach-Salazar Sáenz, Sabine

2008 «Dictionaries, Vocabularies, and Grammars of Andean Indigenous Languages». En: Joanne Pillsbury (ed.). Guide to Documentary Sources for Andean Studies 1530-1900. Vol. 1, pp. 235-264.

2013 Entrelazando dos mundos: Experimentos y experiencias con el quechua de la cristianización en el Perú colonial. Quito: Abya-Yala.

Doesburg, Bas van y Michael W. Swanton

2011 «Mesoamerican Philology as an Interdisciplinary Study: The Chochon (Xru Ngiwa) "Barrios" of Tamazulapan (Oaxaca, Mexico)». Ethnohistory, vol. 58, n. 4, pp. 613-652.

Durston, Alan

2007 Pastoral Quechua: The History of Christian Translation in Colonial Peru, 1550-1650. Notre Dame, IN: University of Notre Dame Press.

2008 «Native-Language Literacy in Colonial Peru: The Question of Mundane Quechua Writing Revisited». Hispanic American Historical Review, vol. 88, n. 1, pp. 41-70.

2011 «Quechua Political Literature in Early Republican Peru (18211876)». En: Paul Heggarty y Adrian Pearce (eds.). History and Language in the Andes. Nueva York: Palgrave Macmillan, pp. 165-186.

2014 «El teatro quechua en la ciudad de Ayacucho, Perú, 1920-1950». En: Corpus: Archivos Virtuales de la Alteridad Americana, vol. 4 n. 2. 
Publicación electrónica [en línea]: <http://corpusarchivos.revues. org/1280>

[En prensa] «Quechua for the Patria Nueva: Indigenous-Language Government Propaganda in 1920s Peru». En: Alan Durston, Sabine MacCormack y Bruce Mannheim (eds.). Authority, Hierarchy, and the Indigenous Languages of Latin America: Historical and Ethnographic Perspectives. University of Notre Dame Press.

EDmonson, Munro S. (trad. y ed.)

1971 The Book of Counsel: The Popul Vuh of the Quiché Maya of Guatemala. Nueva Orleans: Middle American Research Institute, Tulane University.

Edmonson, Munro S. y Patricia A. Andrews (eds.)

1984 Handbook of Middle American Indians. Supplement 4: Linguistics. Austin: University of Texas Press.

1985 Handbook of Middle American Indians. Supplement 2: Literatures. Austin: University of Texas Press.

Estenssoro Fuchs, Juan Carlos

2003 Del paganismo a la santidad: La incorporación de los indios del Perú al catolicismo, 1532-1750. Trad. de Gabriela Ramos. Lima: Instituto Francés de Estudios Andinos y Pontificia Universidad Católica del Perú - Instituto Riva-Agüero.

HANKs, William

2010 Converting Words: Maya in the Age of the Cross. Los Angeles: University of California Press.

HARRISON, Regina

2008 «Doctrinal Works». En: Joanne Pillsbury (ed.). Guide to Documentary Sources for Andean Studies 1530-1900. Vol. 1, pp. 217-234.

2014 Sin and Confession in Colonial Peru: Spanish-Quechua Penitential Texts, 1560-1650. Austin: University of Texas Press.

HarTch, Todd

2006 Missionaries of the State: The Summer Institute of Linguistics, State Formation, and Indigenous Mexico, 1935-1985. Tuscaloosa: University of Alabama Press. 
Heggarty, Paul y Adrian Pearce (eds.)

2011 History and Language in the Andes. Nueva York: Palgrave Macmillan.

Heggarty, Paul y David Beresford-Jones (eds.)

2012 Archaeology and Language in the Andes: A Cross-Disciplinary Exploration of Prehistory. Oxford: Oxford University Press.

ITIER, César

1995a El teatro quechua en el Cuzco. Tomo I. Instituto Francés de Estudios Andinos y Centro de Estudios Regionales Andinos Bartolomé de Las Casas.

1995b «Quechua y cultura en el Cuzco del siglo XvIII: de la "lengua general" al "idioma del imperio de los incas"». En César Itier (ed.), Del siglo de oro al siglo de las luces. Lenguaje y sociedad en los Andes del siglo XVIII. Cusco: Centro de Estudios Regionales Andinos Bartolomé de Las Casas, pp. 90-102.

2000 El teatro quechua en el Curco. Tomo II. Instituto Francés de Estudios Andinos y Centro de Estudios Regionales Andinos Bartolomé de Las Casas.

2002 «Quechua, Aymara and Other Andean Languages: Historical, Linguistic and Sociolinguistic Aspects». En: EnterText: An Interdisciplinary Humanities E-Journal 2.2, pp. 136-165. Publicación electrónica [en línea]: <http://www.brunel.ac.uk/_data/assets/ pdf_file/0013/111172/Cesar-Itier-Quechua,-Aymara-and-otherAndean-Languages-Historical,-Linguistic-and-Socio-linguisticAspects.pdf $>$

2013 «Las bases geográficas de la lengua vehicular del imperio inca». Bulletin de l'Institut Français d'Études Andines, vol. 42, n. 2, pp. 237-260.

ITIER, César y Capucine Boidin

2013 «Presentación general de Les langues générales d'Amérique du sud (XVIXIX)». En: Nuevo Mundo Mundos Nuevos. Publicación electrónica [en línea]: <http://nuevomundo.revues.org/65045>

Itier, César, Luis Nieto Degregori y Jorge Alejandro Vargas Prado (comp.) 2012 Qosqo qhechwasimipi akllasqa rimaykuna. Antología quechua del Cusco. Cusco: Municipalidad Provincial del Cusco. 
KeLlogg, Susan

1995 Law and The Transformation of Aztec Culture, 1500-1700. Norman: University of Oklahoma Press.

Kerke, Simon van y Pieter Muysken

2014 «The Andean Matrix». En: O’Connor and Muysken (eds). The Native Languages of South America: Origins, Development, Typology. Cambridge y Nueva York: Cambridge University Press, pp. 126-151.

Laughlin, Robert M.

2003 Beware the Great Horned Serpent!: Chiapas under the Threat of Napoleon. Albany NY: Institute for Mesoamerican Studies, University at Albany.

LeE, M. Kittiya

2014 «Language and Conquest: Tupi-Guarani Expansion in the European Colonization of Brazil and Amazonia». En: Salikoko Mufwene (ed.). Iberian Imperialism and Language Evolution in Latin America. Chicago: The University of Chicago Press, pp. 143-167.

LeINHARD, Martin

1992 La voz y su buella. Escritura y conflicto étnico-cultural en América Latina 1492-1988. 3. ${ }^{a}$ ed. Lima: Horizonte.

LeÓN-Portilla, Miguel

1959 Visión de los vencidos: Relaciones indígenas de la conquista. Ciudad de México: Universidad Nacional Autónoma de México.

1978 Los manifiestos en nábuatl de Emiliano Zapata. Ciudad de México: Universidad Nacional Autónoma de México, Instituto de Investigaciones Históricas.

LOCKHART, James

1991 Nabuas and Spaniards: Postconquest Central Mexican History and Pbilology. Stanford y Los Ángeles: Stanford University Press y UCLA Latin American Center Publications.

1992 The Nahuas after the Conquest: A Social and Cultural History of the Indians of Central Mexico, Sixteenth through Eighteenth Centuries. Stanford: Stanford University Press. 
1997 «Introduction: Background and Course of the New Philology». En: J. Lockhart, L. Sousa y S. Wood (eds.). Sources and Methods for the Study of Postconquest Mesoamerican Ethnohistory, pp. 1-24. Publicación electrónica [en línea]: <http://whp.uoregon.edu/Lockhart/>

Lockhart, James, Frances Berdan y Arthur J. O. Anderson (eds.)

1986 The Tlaxcalan Actas: A Compendium of the Records of the Cabildo of Tlaxcala (1545-1627). Salt Lake City: University of Utah Press.

LockharT, James, Lisa Sousa y Stephanie Wood (eds.)

2007 Sources and Methods for the Study of Postconquest Mesoamerican Ethnohistory. Publicación electrónica [en línea]: <http://whp.uoregon. edu/Lockhart/>

Lustig, Wolf

2007 «El guaraní lengua de guerreros? La “raza guaranî” y el avañe'e en el discurso bélico-nacionalista del Paraguay». En: Nicolas Richard et ál. (eds.). Les guerres du Paraguay aux XIXE et XXE siècles. París: CoLibris, pp. 525-540.

MacCormack, Sabine

1985 "The Heart Has Its Reasons": Predicaments of Missionary Christianity in Early Colonial Peru». Hispanic American Historical Review, vol. 65, n. 3, pp. 443-466.

Mallon, Florencia

2012 Decolonizing Native Histories: Collaboration, Knowledge, and Language in the Americas. Durham y Londres: Duke University Press.

Mannheim, Bruce

1991 The Language of the Inka since the European Invasion. Austin: University of Texas Press.

MELIÀ, Bartomeu

1992 La lengua guaraní del Paraguay: Historia, sociedad y literatura. Madrid: MAPFRE.

2003 La lengua guarani en el Paraguay colonial, que contiene la creación de un lenguaje cristiano en las reducciones de los guaranies en el Paraguay. Asunción: CEPAG. 
2011 «La lengua guaraní dependiente en tiempos de independencia en Paraguay». Journal de la Société des Américanistes, vol. 97, n. 2, pp. 153174.

Moore, Denny

2014 «Historical Development of Nheengatu (Língua Geral Amazônica)». En: Salikoko Mufwene (ed.). Iberian Imperialism and Language Evolution in Latin America. Chicago: The University of Chicago Press, pp. 108-142.

MORRIs, Mark

2007 «Language in Service of the State: The Nahuatl Conterinsurgency Broadsides of 1810». Hispanic American Historical Review, vol. 87, n. 3, pp. 433-470.

Mufwene, Salikoko (ed.)

2014 Iberian Imperialism and Language Evolution in Latin America. Chicago: The University of Chicago Press.

Neumann, Eduardo S.

2007 «A escrita dos guaranis nas reduções: usos e funções das formas textuais indígenas, século xvIII». Topoi, n. 8, pp. 48-79.

2014 «A escrita em guarani dos mayordomos e o exemplo de Valentín Ybariguá (Paraguai, XVIII)». En: Corpus: Archivos Virtuales de la Alteridad Americana, vol. 4, n. 2. Publicación electrónica [en línea]: http://corpusarchivos.revues.org/1258

O'Connor, L. y Pieter C. Muysken

2014 The Native Languages of South America: Origins, Development, Typology. Cambridge y Nueva York: Cambridge University Press.

Pavez OjedA, Jorge

2003 «Mapuche ñi nutram chilkatun. Escribir la historia mapuche: Estudio poslaminar de Kiñe mufu trokinche ñi piel. Historias de familias. Siglo XIX». Revista de Historia Indigena, n. 7, pp. 7-53.

Pearce, Adrian

2011 «Reindigenization and Native Languages in Peru's Long Nineteenth Century (1795-1940)». En: Paul Heggarty y Adrian Pearce 
(eds.). History and Language in the Andes. Nueva York: Palgrave Macmillan, pp. 135-164.

Pillsbury, Joanne (ed.)

2008 Guide to Documentary Sources for Andean Studies 1530-1900. 3 vols. Norman: University of Oklahoma Press.

PizZigoni, Caterina

2012 The Life Within: Local Indigenous Society in Mexico's Toluca Valley, 1650-1800. Stanford: Stanford University Press.

QuiLTER, Jeffrey y Gary Urton (eds.)

2002 Narrative Threads: Accounting and Recounting in Andean Khipu. Austin: University of Texas Press.

Restall, Matthew

1997 The Maya World. Yucatec Culture and Society, 1550-1850. Stanford: Stanford University Press.

2003 «A History of the New Philology and the New Philology in History». Latin American Research Review, vol. 38, n. 1, pp. 113-134.

Restall, Matthew, Lisa Sousa y Kevin Terraciano (eds.)

2005 Mesoamerican Voices: Native-Language Writings from Colonial Mexico, Oaxaca, Yucatan and Guatemala. Cambridge y Nueva York: Cambridge University Press.

Roys, Ralph L. (ed. y trad.)

1933 The Book of Chilam Balam of Chumayel. Washington: Carnegie Institution.

SAHAGÚn, Bernardino de

1950-1982 Florentine Codex: General History of the Things of New Spain. 12 vols. Trad. y ed. por Charles E. Dibble y Arthur J. O. Anderson. Salt Lake City: University of Utah Press.

SALOMON, Frank

1999 «Testimonies: The Making and Reading of Native South American Historical Sources». En: Frank Salomon y Stuart B. Schwartz (eds.). The Cambridge History of the Native Peoples of the Americas. Vol. 3: South America. Cambridge: Cambridge University Press, pp. 19-95. 
2004 The Cord Keepers: Khipus and Cultural Life in a Peruvian Village. Durham: Duke University Press.

SALOMON, Frank y George L. Urioste

1991 The Huarochiri Manuscript: A Testament of Ancient and Colonial Andean Religion. Austin: University of Texas Press.

Sigal, Pete

2000 From Moon Goddesses to Virgins: The Colonization of Yucatecan Maya Sexual Desire. Austin: University of Texas Press.

2011 The Flower and the Scorpion: Sexuality and Ritual in Early Nahua Culture. Durham, NC: Duke University Press.

Solano, Francisco de

1991 Documentos sobre política lingüistica en Hispanoamérica (1492-1800).

Madrid: Consejo Superior de Investigaciones Científicas.

STANFIELD-Mazzi, Maya

2013 Object and Apparition: Envisioning the Christian Divine in the Colonial Andes. Tucson: University of Arizona Press.

SwANTON, Michael

2008 «Multilingualism in the Tocuij Nudzavui Region». En: Maarten E. G. R. N. Jansen y Laura N. K. van Broekhoven (eds.). Mixtec Writing and Society: Escritura de Nun Draui. Amsterdam: Koninlijke Nederlandse Akademie van Wetenschappen, pp. 347-380.

TAYLOR, Gerald

2000 Camac, camay y camasca y otros ensayos sobre Huarochiri y Yauyos. Lima: Instituto Francés de Estudios Andinos y Centro de Estudios Regionales Andinos Bartolomé de Las Casas.

2003 El sol, la luna y las estrellas no son Dios: La evangelización en quechua (siglo XVI). Lima: Instituto Francés de Estudios Andinos y Pontificia Universidad Católica del Perú.

TAYLOR, Gerald (ed.)

1999 Ritos y tradiciones de Huarochirí: Segunda edición revisada. Lima: Instituto Francés de Estudios Andinos, Banco Central de Reserva del Perú y Universidad Ricardo Palma. 
TAVÁREZ, David

2011 The Invisible War: Indigenous Devotions, Discipline, and Dissent in Colonial Mexico. Stanford: Stanford University Press.

Tedlock, Dennis

20102000 Years of Mayan Literature. Berkeley: University of California Press.

Terraciano, Kevin

2001 The Mixtecs of Colonial Oaxaca. Nudzabui History, Sixteenth through Eighteenth Centuries. Stanford: Stanford University Press.

Torero, Alfredo

1974 El quechua y la historia social andina. Lima: Universidad Ricardo Palma.

Townsend, Camilla (ed. y trad.)

2010 Here in This Year: Seventeenth-Century Nabuatl Annals of the TlaxcalaPuebla Valley. Stanford: Stanford University Press.

Whigham, Thomas

2012 «Building the Nation While Destroying the Land: Paraguayan Journalism during the Triple Alliance War, 1864-1870». Jabrbuch für Geschichte Lateinamerikas, n. 49, pp. 157-180.

YANNAKAKIS, Yanna

2012 «Introduction: How Did They Talk to One Another? Language Use and Communication in Multilingual New Spain». Ethnohistory, vol. 59, n. 4, pp. 667-674.

YannAKakis, Yanna y Martina Schrader-Kniffki

2014 «Sins and Crimes: Zapotec-Spanish Translation in Catholic Evangelization and Colonial Law in Oaxaca, New Spain». En: Klaus Zimmerman, Martina Schrader-Kniffki y Otto Zwartjes (eds.). Missionary Linguistics V. Lingüistica Misionera V: Translation Theories and Practices. Amsterdan: John Benjamins, pp. 161-199. 